Communications in Physics, Vol.23, No.1 (2013), pp. 29-37

\title{
ON THE COHERENT STATE METHOD IN CONSTRUCTING REPRESENTATIONS OF QUANTUM SUPERALGEBRAS
}

\author{
NGUYEN CONG KIEN \\ Institute of Physics, VAST \\ NGUYEN ANH KY \\ Institute of Physics, VAST \\ and \\ Laboratory for High Energy Physics and Cosmology, \\ Faculty of Physics, College of Science, Vietnam National University, Hanoi
}

\begin{abstract}
In recent years, one of the new applications of the coherent state method was to construct representation of superalgebras and quantum superalgebras. Following this stream, we had a contribution to working out explicit representation of $U_{q}[g l(2 \mid 1)]$. Up to now, $U_{q}[g l(2 \mid 1)$ is still the biggest quantum superalgebra representations in coherent state basis of which can be built. In this article, we will show some detailed techniques used in our previous work but useful for our further investigations. The newest results on building representations in a coherent state basis of $U_{q}[\operatorname{osp}(2 \mid 2)]$, which has the same rank as $U_{q}[g l(2 \mid 1)]$, are also briefly exposed.
\end{abstract}

\section{INTRODUCTION}

In the late 1920's, the concept of coherent states (CS's) was introduced by E. Schrödinger [1] while searching for a classical analog of quantum states of quantum harmonic oscillators. For more than 80 years, the concept of CS's has been developed by many people, especially, a crutial step was made by A. Perelomov who generalized the CS concept for arbitrary Lie algebras [2-4]. This concept was also extended to that of vector coherent states (VCS's) [5-9]. In 1970's, the formation of supersymmetry (SUSY) led to the creation of a new research trend in physics and mathematics (although, presently, the SUSY phenomenological models are in a difficult time when the latest results of the LHC have not been able to confirm them). Combining with the SUSY idea, the concept of CS's was developed to those of super coherent states (SCS's) and supervector coherent states (SVCS's) [10-14].

With special characteristics, CS's are quantum states having a direct relation to classical states (see [15] and references therein). Therefore, it has been applied to a wide range of various fields from quantum optics [16], quantum information [17], condensed matter physics [4], string theories [18,19] and cosmology [20]. The coherent state method is also very useful when we utilize them to construct representations of Lie (super) groups and algebras (see, for example, [24] and refereces therein).

For the last time, some authors have started exploiting the CS method to build finite dimensional representations of superalgebras and quantum superalgebras. However, 
they have succeeded with only a few low-rank superalgebras and the simplest quantum superalgebra $U_{q}[\operatorname{osp}(1 \mid 2)]$ (see $[21,22]$ ). To use this method for higher rank quantum superalgebras, e.g., $U_{q}[g l(2 \mid 1)]$, we developed calculating procedure that concerns high order commutator expressions and $q$-calculus [24]. In this paper, we show them in details with a new application to the construction of boson-fermion representations of $U_{q}[\operatorname{sosp}(2 \mid 2)]$. For the convenience of the reader, nonetheless, we recall first the results obtained in the case of $U_{q}[g l(2 \mid 1)]$ before considering $U_{q}[\operatorname{osp}(2 \mid 2)]$. Some of the present results were reported at the recent national meeting in theoretical physics [25].

\section{SOME IMPORTANT TECHNIQUE OF CONSTRUCTING REPRESENTATIONS OF QUANTUM GROUP BY COHERENT STATE METHOD}

When we work with quantum (super) algebras, most of transformations are related with deformation parameters. In this paper, to be simple, we only mention quantum (super) algebras with one deformation parameter which will be denoted here by $q$.

Suppose $X$ is an arbitrary number or an operator, we define the $q$-deformation $[X]_{q}$ and the $q$-expression $\{X\}_{q}$ :

$$
\begin{aligned}
& {[X]_{q}=\frac{q^{X}-q^{-X}}{q-q^{-1}}} \\
& \{X\}_{q}=q^{X}+q^{-X} .
\end{aligned}
$$

and $q$-exponent:

$$
e_{q}^{X}=\sum_{n=0}^{\infty} \frac{X^{n}}{[n]_{q} !} .
$$

with $n$ is an integer and $[n]_{q} !=[1]_{q}[2]_{q} \ldots[n]_{q}$. Basically, other trancendental functions that includes $q$-parameter can be represented through normal polynomials by Taylor's expansion.

Now, with operators $X$ and $Y$, we can define deformed (anti) commutators:

$$
\begin{aligned}
{[X, Y]_{q} } & =X Y-q Y X \\
\{X, Y\}_{q} & =X Y+q Y X .
\end{aligned}
$$

Here we list some expressions,

$$
\begin{aligned}
{[X+2]_{q}-[X]_{q} } & =\{X+1\}_{q}, \\
\{X+Y\}_{q}+\{X-Y\}_{q} & =\{X\}_{q}\{Y\}_{q}, \\
\{X+Y\}_{q}-\{X-Y\}_{q} & =\left(q-q^{-1}\right)^{2}[X]_{q}[Y]_{q}, \\
{[X+Y]_{q}+[X-Y]_{q} } & =[X]_{q}\{Y\}_{q}, \\
{[X+Y]_{q}-[X-Y]_{q} } & =[Y]_{q}\{X\}_{q} .
\end{aligned}
$$




$$
\begin{aligned}
\sum_{i=0}^{n-1} q^{2 i} & =q^{n-1}[n]_{q} \\
\sum_{i=0}^{n-1} q^{-2 i} & =q^{1-n}[n]_{q} \\
\sum_{i=0}^{n-1} q^{4 i} & =q^{2 n-2}[n]_{q} \frac{\{n\}_{q}}{\{1\}_{q}}, \\
\sum_{i=0}^{n-1} q^{-4 i} & =q^{-2 n+2}[n]_{q} \frac{\{n\}_{q}}{\{1\}_{q}} \\
\sum_{i=0}^{n-1}\{X-2 i\}_{q} & =\{X-n+1\}_{q}[n]_{q}, \\
\sum_{i=0}^{n-1}[X-2 i]_{q}\{Y-2 i\}_{q} & =[X+Y-2 n+2]_{q}[n]_{q} \frac{\{n\}_{q}}{\{1\}_{q}}+n[X-Y]_{q}, \\
\sum_{i=0}^{n-1}\{X-i\}_{q}[i]_{q}\{Y-2 i\}_{q} & =\left(\begin{array}{l}
{[X+Y-n+1]_{q}+[X-Y+n-1]_{q}} \\
-[X+Y-2 n+2] \frac{\{n\}_{q}}{\{1\}_{q}}-\frac{n}{[n]_{q}}[X-Y]_{q}
\end{array}\right)[n]_{q},
\end{aligned}
$$

which are often used.

\section{REPRESENTATION OF $U_{q}[o s p(2 \mid 2)]$ IN A COHERENT STATE BASIS}

In this section, we recall some elements of the algebraic structure and representations in a CS basis of $U_{q}[g l(2 \mid 1)]$ (see [24]), a quantum superalgebra which has been invetigated in details by both physicists and mathematicians. Next, we will show how to establish the $U_{q}[g l(2 \mid 1)]$ coherent states that will be used to construct its representations of this quantum superalgebra.

\section{III.1. Quantum superalgebra $U_{q}[g l(2 \mid 1)]$}

The quantum super algebra $U_{q}[g l(2 \mid 1)]$ can be defined through its Weyl-Chevalley generators $E_{12}, E_{21}, E_{23}, E_{32}$ and $E_{i i}(i=1,2,3)$ satisfying the following defining relations (see, for example, $[23,24])$ :

a) the (anti-)commutation relations $(1 \leq i, j, i+1, j+1 \leq 3)$ :

$$
\begin{gathered}
{\left[E_{i i}, E_{j j}\right]=0,} \\
{\left[E_{i i}, E_{j, j+1}\right]=\left(\delta_{i j}-\delta_{i, j+1}\right) E_{j, j+1},} \\
{\left[E_{i i}, E_{j+1, j}\right]=\left(\delta_{i, j+1}-\delta_{i, j}\right) E_{j+1, j},} \\
{\left[E_{12}, E_{21}\right]=\left[H_{1}\right]_{q},} \\
\left\{E_{23}, E_{32}\right\}=\left[H_{2}\right]_{q}, \\
H_{i}=\left(E_{i i}-\frac{d_{i+1}}{d_{i}} E_{i+1, i+1}\right), H_{3} \equiv E_{33},
\end{gathered}
$$


where $d_{1}=d_{2}=-d_{3}=1$, and

b) the Serre relations:

$$
\begin{aligned}
E_{23}^{2} & =E_{32}^{2}=0, \\
{\left[E_{12}, E_{13}\right]_{q} } & =\left[E_{21}, E_{31}\right]_{q}=0,
\end{aligned}
$$

with $E_{13}$ and $E_{31}$,

$$
E_{13}=\left[E_{12}, E_{23}\right]_{q^{-1}}, E_{31}=-\left[E_{21}, E_{32}\right]_{q^{-1}} .
$$

The generators $E_{i j}, i, j=1,2,3$, are $q$-deformations ( $q$-analogs) of the corresponding Weyl generators $e_{i j}$ of classical super-algebra $g l(2 \mid 1)$ obeying the (anti) commutation relations

$$
\left[e_{i j}, e_{k l}\right\}=\delta_{j k} e_{i l}-(-1)^{([i]+[j])([k]+[l])} \delta_{i l} e_{k j},
$$

with $\left[e_{i j}, e_{k l}\right\} \equiv e_{i j} e_{k l}-(-1)^{([i]+[j])([k]+[l])} e_{k l} e_{i j}$, and [i] being a parity defined by $[1]=[2]=$ $0,[3]=1$. It is easy to see that $H_{i}$ and the even Chevalley generators $E_{12}$ and $E_{21}$ form a subalgebra called the even subalgebra of $U_{q}[g l(2 \mid 1)]$ and denoted by $U_{q}\left[g l(2 \mid 1)_{\overline{0}}\right]$. The latter is a quantum deformation of $U\left[g l(2 \mid 1)_{\overline{0}}\right]$ which is the universal enveloping algebra of the even subalgebra $g l(2 \mid 1)_{\overline{0}}$ of $g l(2 \mid 1)$.

\section{III.2. Coherent states of $U_{q}[g l(2 \mid 1)]$}

Now, we elaborate the vector coherent states of $U_{q}[g l(2 \mid 1)]$ in a basis of an elementary representation and the Fock-space of the corresponding quantum superalgebra Heisenberg.

Let $|J\rangle$ be a state of $U_{q}[g l(2 \mid 1)]$ defined by

$$
\begin{gathered}
H_{1}|J\rangle=2 J_{1}|J\rangle, H_{2}|J\rangle=2 J_{2}|J\rangle, H_{3}|J\rangle=2 J_{3}|J\rangle, \\
E_{12}|J\rangle=E_{13}|J\rangle=E_{23}|J\rangle=0 .
\end{gathered}
$$

It is called the the highest weight state characterized by a set of three numbers $J_{1}, J_{2}$ and $J_{3}$ called the highest weight:

$$
|J\rangle \equiv\left|J_{1}, J_{2}, J_{3}\right\rangle \text {. }
$$

If we want an elementary reprenstation of $U_{q}[g l(2 \mid 1)]$ finite dimensional, $J_{1}$ must be an integer or a half-integer and in this case the representation space (module), we denote by $W$, has the dimension $8 J_{1}+4$. In particular, it contains four sub-spaces, say $V_{i}$, $i=1,2,3,4$, which are finite-dimensional modules of the even subalgebra $U_{q}\left[g l(2 \mid 1)_{\overline{0}}\right]$ and respectively spanned on the following basis vectors:

$$
\begin{aligned}
\left|J_{1}, J_{2}, J_{3}, M\right\rangle= & C_{M}^{(1)} \cdot E_{21}^{J_{1}-M}|J\rangle \in V_{1}, \\
\left|J_{1}-1 / 2, J_{2}+1 / 2, J_{3}+1 / 2, P\right\rangle= & C_{P}^{(2)} \cdot\left\{q^{J_{1}+P+1 / 2} E_{32} E_{21}^{J_{1}-P+1 / 2}|J\rangle\right. \\
& \left.+\left[J_{1}+P+1 / 2\right]_{q} E_{31} E_{21}^{J_{1}-P-1 / 2}|J\rangle\right\} \in V_{2}, \\
\left|J_{1}+1 / 2, J_{2}, J_{3}+1 / 2, R\right\rangle= & C_{R}^{(3)} \cdot\left\{\frac{1}{q^{J_{1}-R+1 / 2}} E_{32} E_{21}^{J_{1}-R+1 / 2}|J\rangle\right. \\
& \left.-\left[J_{1}-R+1 / 2\right]_{q} E_{31} E_{21}^{J_{1}-R-1 / 2}|J\rangle\right\} \in V_{3}, \\
\left|J_{1}, J_{2}+1 / 2, J_{3}+1, S\right\rangle= & C_{S}^{(4)} \cdot E_{32} E_{31} E_{21}^{J_{1}-S}|J\rangle \in V_{4},
\end{aligned}
$$


where $C_{K}^{(i)}$ are normalization coefficients which can be fixed by addtional, for example, Hermitian conditions, and $-J_{1} \leq M, S \leq J_{1},-\left(J_{1}-1 / 2\right) \leq P \leq\left(J_{1}-1 / 2\right),-\left(J_{1}+1 / 2\right) \leq$ $R \leq\left(J_{1}+1 / 2\right)$ such that $\left(J_{1}-M\right),\left(J_{1}-P-1 / 2\right),\left(J_{1}-R+1 / 2\right)$ and $\left(J_{1}-S\right)$ are integers. These four finite-dimensional irreducible modules of the even subalgebra $U_{q}[g l(2 \mid 1) \overline{0}]$ are built on the $U_{q}\left[g l(2 \mid 1)_{\overline{0}}\right]$-highest weight states

$$
\begin{aligned}
\left|J_{1}, J_{2}, J_{3} ; J_{1}\right\rangle & \equiv\left|J_{1}, J_{2}, J_{3}\right\rangle=|J\rangle, \\
\left|J_{1}-1 / 2, J_{2}+1 / 2, J_{3}+1 / 2 ; J_{1}-1 / 2\right\rangle & \equiv\left|J_{1}-1 / 2, J_{2}+1 / 2, J_{3}+1 / 2\right\rangle, \\
\left|J_{1}+1 / 2, J_{2}, J_{3}+1 / 2 ; J_{1}+1 / 2\right\rangle & \equiv\left|J_{1}+1 / 2, J_{2}, J_{3}+1 / 2\right\rangle, \\
\left|J_{1}, J_{2}+1 / 2, J_{3}+1 ; J_{1}\right\rangle & \equiv\left|J_{1}, J_{2}+1 / 2, J_{3}+1\right\rangle,
\end{aligned}
$$

via the formula

$$
\left|L_{1}, L_{2}, L_{3} ; k\right\rangle=C_{k}^{(i)} \cdot\left(E_{21}\right)^{L_{1}-k}\left|L_{1}, L_{2}, L_{3} ; L_{1}\right\rangle, i=1,2,3,4,
$$

where $C_{k}^{(i)}$ are normalization coefficients in (12), $L_{1}=J_{1}, J_{1} \pm 1 / 2 ; L_{2}=J_{2}, J_{2}+1 / 2$; $L_{3}=J_{3}, J_{3} \pm 1 / 2, J_{3}+1$ and $-L_{1} \leq k \leq L_{1}$ such that $L_{1}-k$ are integers. For simplicity, we can choose $C_{k}^{(i)}=1$ and work with vectors (12) non-normalized. The odd generators of $U_{q}\left[g l(2 \mid 1)\right.$ intertwine these $U_{q}\left[g l(2 \mid 1)_{\overline{0}}\right]$-modules. $[10]-[14])$

Now, the generalized coherent states of $U_{q}[g l(2 \mid 1)]$ can be defined as (cf. $[3,4,21]$,

$$
e_{q}^{a_{12} E_{21}+\alpha_{23} E_{32}+\alpha_{13} E_{31}}|J\rangle,
$$

where $\alpha_{i j}^{\dagger}\left(\alpha_{i j}\right)$ are $q$-analogs of the fermion creating (annihilating) operators with the number operator $N_{\alpha_{i j}}$, and $a_{12}^{\dagger}\left(a_{12}\right)$ is a $q$-analog of the boson creating (annihilating) operator with the number operator $N_{a_{12}}$. This operators form the quantum Heisenberg superalgebra $U_{q}[h(2 \mid 1)]$ :

$$
\begin{array}{r}
\left\{\alpha_{i j}, \alpha_{i j}^{\dagger}\right\}=1, N_{\alpha_{i j}}=\alpha_{i j}^{\dagger} \alpha_{i j},\left[N_{\alpha_{i j}}, \alpha_{i j}^{\dagger}\right]=\alpha_{i j}^{\dagger},\left[N_{\alpha_{i j}}, \alpha_{i j}\right]=-\alpha_{i j} \\
{\left[a_{12}, a_{12}^{\dagger}\right]_{q}=q^{-N_{a_{12}}},\left[N_{a_{12}}\right]_{q}=a_{12}^{\dagger} a_{12},\left[N_{a_{12}}+1\right]_{q}=a_{12} a_{12}^{\dagger},} \\
{\left[N_{a_{12}}, a_{12}^{\dagger}\right]=a_{12}^{\dagger},\left[N_{a_{12}}, a_{12}\right]=-a_{12} .}
\end{array}
$$

This quantum Heisenberg superalgebra $U_{q}[h(2 \mid 1)]$ super-commutes with $U_{q}[g l(2 \mid 1)]$, that is, if $E$ is an operator of $U_{q}[g l(2 \mid 1)]$ and $X$ is an operator of $U_{q}[h(2 \mid 1)]$ they super-commute with each other:

$$
E X=(-1)^{\operatorname{deg}(E) \cdot \operatorname{deg}(X)} X E,
$$

where $\operatorname{deg}(X)$ is the parity of $X$.

Let $|\psi\rangle$ be a state vector in a (e.g., finite-dimensional) module of $U_{q}[g l(2 \mid 1)]$. Then the mapping [24] (note the difference between the notations here and [21])

$$
|\psi\rangle \rightarrow|\psi\rangle_{J}=\left\langle J\left|e_{q}^{a_{12}^{\dagger} E_{12}+\alpha_{23}^{\dagger} E_{23}+\alpha_{13}^{\dagger} E_{13}}\right| \psi\right\rangle|0\rangle,
$$


induces the mapping

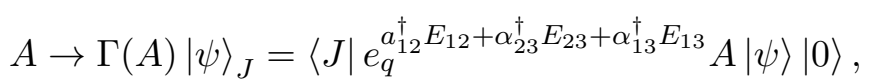

of an operator $A$ defined in a space, which here is a $U_{q}[g l(2 \mid 1)]$ module, containing $|\psi\rangle$, where $|0\rangle$ is a vacuum state of the quantum Heisenberg superalgebra $U_{q}[h(2 \mid 1)$ :

$$
a_{12}|0\rangle=\alpha_{i j}|0\rangle=0 .
$$

The main purpose of coherent state method is to find $|\psi\rangle_{J}$ and explicit forms of $\Gamma(A)$. The explicit results can be found in [24].

\section{REPRESENTATION OF $U_{q}[o s p(2 \mid 2)]$ IN A COHERENT STATE BASIS}

Here, we consider $U_{q}[\operatorname{osp}(2 \mid 2)]$ and first see how its algebraic structure is broken in the deformation process from $\operatorname{osp}(2 \mid 2)$. The rest part is to construct boson-fermion realizations (finite-dimensional representations) of $U_{q}[\operatorname{osp}(2 \mid 2)]$.

IV.1. Quantum superalgebra $U_{q}[\operatorname{osp}(2 \mid 2)]$

Quantum superalgebra $U_{q}[\operatorname{osp}(2 \mid 2)]$ can be defined via its Weyl-Chevalley generators

$E, F, e, \bar{e}, f, \bar{f}$ which satisfy the following defining relations (see, for example, [26]):

$$
\begin{array}{r}
\{e, f\}=-\frac{1}{2}\left[H_{1}-H_{2}\right]_{q}, \quad\{\bar{e}, \bar{f}\}=-\frac{1}{2}\left[H_{1}+H_{2}\right]_{q} \\
{\left[H_{1}, e\right]=e,\left[H_{1}, f\right]=-f,\left[H_{2}, e\right]=e,\left[H_{2}, f\right]=-f,} \\
{\left[H_{1}, \bar{e}\right]=\bar{e},\left[H_{1}, \bar{f}\right]=-\bar{f},\left[H_{2}, \bar{e}\right]=-\bar{e}\left[H_{2}, \bar{f}\right]=\bar{f}} \\
\{e, \bar{e}\}=E,\{f \bar{f}\}=-F,\left[H_{1}, E\right]=2 E,\left[H_{1}, F\right]=-2 F, \\
{[E, F]=-\frac{1}{2} e\left\{H_{1}+H_{2}-1\right\}_{q}-\frac{1}{2} f e\left\{H_{1}+H_{2}+\right\}_{q}} \\
-\frac{1}{2} \bar{e} \bar{f}\left\{H_{1}-H_{2}-1\right\}_{q}-\frac{1}{2} \bar{f} \bar{e}\left\{H_{1}-H_{2}+\right\}_{q} \\
{[E, f]=-\frac{1}{2} \bar{e}\left\{H_{1}-H_{2}+1\right\}_{q},[E, \bar{f}]=-\frac{1}{2} e\left\{H_{1}+H_{2}+1\right\}_{q}} \\
{[F, e]=-\frac{1}{2} \bar{f}\left\{H_{1}-H_{2}-1\right\}_{q},[F, \bar{e}]=-\frac{1}{2} f\left\{H_{1}+H_{2}-1\right\}_{q} .}
\end{array}
$$

Let us note a feature related to a quantum deformation of an orthosymplectic superalgerbra. In classical case, algebraically, three generators $H_{1}, E$, and $F$ generate a subagebra $s u(2)$ of $\operatorname{ssp}(2 \mid 2)$. However when we deform this superalgebra to get its quantum version - $U_{q}[\operatorname{osp}(2 \mid 2)]$, as above, the subalgebra structure is broken. In orther words, now $H_{1}, E$ and $F$ do not form a quantum algebra $U_{q}[s u(2)]$.

\section{IV.2. q-boson-fermion realization of $U_{q}[\operatorname{ssp}(2 \mid 2)]$}

In this sub-section, we deal with $q$-boson-fermion realizations of $U_{q}[\operatorname{osp}(2 \mid 2)]$. As shown above, $U_{q}[\operatorname{osp}(2 \mid 2)]$ does not contain a subalgebra $U_{q}[s u(2)]$, therefore, building finite-dimensional represenations of $U_{q}[\operatorname{osp}(2 \mid 2)]$ based on an even subalgebra is impossible. To solve this problem, we start from a state, say $|J\rangle$, defined by the conditions

$$
H_{1}|J\rangle=2 J_{1}|J\rangle, H_{2}|J\rangle=2 J_{2}|J\rangle, E|J\rangle=e|J\rangle=\bar{e}|J\rangle=0 .
$$


We call it the highest weight state of $U_{q}[\operatorname{osp}(2 \mid 2)]$. We can also define its lowest weight state, $\left|J^{\prime}\right\rangle$ :

$$
H_{1}\left|J^{\prime}\right\rangle=2 J_{1}^{\prime}\left|J^{\prime}\right\rangle, H_{2}\left|J^{\prime}\right\rangle=2 J_{2}^{\prime}\left|J^{\prime}\right\rangle, F\left|J^{\prime}\right\rangle=f\left|J^{\prime}\right\rangle=\bar{f}\left|J^{\prime}\right\rangle=0 .
$$

The lowest weight and the highest one are related to each other through the formula

$$
F^{d}|J\rangle=\left|J^{\prime}\right\rangle
$$

with $n$ is an integer depending on the choice of $J_{i}$ and $J_{i}^{\prime}$. Because the subalgebra structure is broken $J_{1}, J_{1}^{\prime}, J_{2}$ and $J_{2}^{\prime}$ can be arbitrary compex numbers. Using the notation

$$
|J\rangle \equiv\left|J_{1}, J_{2}\right\rangle
$$

the basis vectors on which a representation of $U_{q}[\operatorname{osp}(2 \mid 2)]$ can be spanned can be expressed as

$$
\begin{aligned}
\left|v_{1(n)}\right\rangle & =F^{n}|J\rangle, \\
\left|v_{2(n)}\right\rangle & =f F^{n}|J\rangle, \\
\left|v_{3(n)}\right\rangle & =\bar{f} F^{n}|J\rangle, \\
\left|v_{4(n)}\right\rangle & =\bar{f} f F^{n}|J\rangle .
\end{aligned}
$$

Now, generalized coherent states of $U_{q}[\operatorname{osp}(2 \mid 2)]$ can be defined as (cf. [3,4,21], [10]-

$$
e_{q}^{a F+\alpha_{1} f+\alpha_{2} \bar{f}}|J\rangle
$$

with $\alpha_{i}^{\dagger}\left(\alpha_{i}\right)$ are $q$-analogs of the fermion creating (annihilating) operators with the number operator $N_{\alpha_{i}}$, and $a^{\dagger}(a)$ is a $q$-analog of the boson creating (annihilating) operator with the number operator $N_{a}$. They form the quantum Heisenberg superalgebra $U_{q}[h(2 \mid 1)]$ :

$$
\begin{array}{r}
\left\{\alpha_{i}, \alpha_{i}^{\dagger}\right\}=1, N_{\alpha_{i}}=\alpha_{i}^{\dagger} \alpha_{i},\left[N_{\alpha_{i}}, \alpha_{i}^{\dagger}\right]=\alpha_{i}^{\dagger},\left[N_{\alpha_{i}}, \alpha_{i}\right]=-\alpha_{i} \\
{\left[a, a^{\dagger}\right]_{q}=q^{-N_{a}},\left[N_{a}\right]_{q}=a^{\dagger} a,\left[N_{a}+1\right]_{q}=a a^{\dagger}} \\
{\left[N_{a}, a^{\dagger}\right]=a^{\dagger},\left[N_{a}, a\right]=-a .}
\end{array}
$$

Furthermore, the same as in the case of $U_{q}\left[g l(2 \mid 1)\right.$, if $A$ is an operator of $U_{q}[\operatorname{osp}(2 \mid 2)]$ and $X$ is an operator of $U_{q}[h(2 \mid 1)]$, then:

$$
A X=(-1)^{\operatorname{deg}(A) \cdot \operatorname{deg}(X)} X A
$$

Let $|\psi\rangle$ be a state vector in an elementary representation space of $U_{q}[\operatorname{osp}(2 \mid 2)]$ then the mapping

$$
|\psi\rangle \rightarrow|\psi\rangle_{J}=\left\langle J\left|e_{q}^{a^{\dagger} E+\alpha_{1}^{\dagger} e+\alpha_{2}^{\dagger} \bar{e}}\right| \psi\right\rangle|0\rangle
$$

induces the mapping acts on an operator $\mathrm{A}$ in elementary representation space

$$
A \rightarrow \Gamma(A)|\psi\rangle_{J}=\left\langle J\left|e_{q}^{a^{\dagger} E+\alpha_{1}^{\dagger} e+\alpha_{2}^{\dagger} \bar{e}} A\right| \psi\right\rangle|0\rangle,
$$

where $|0\rangle$ is the vacuum state of the quantum Heisenberg superalgebra $U_{q}[h(2 \mid 1)$ :

$$
a|0\rangle=\alpha_{i}|0\rangle=0 \text {. }
$$


Using (29), we get the following explicit forms of the generators in the coherent state space:

$$
\begin{aligned}
& \Gamma\left(H_{1}\right)=2 J_{1}-2 N_{a}-N_{1}-N_{2}, \\
& \Gamma\left(H_{2}\right)=2 J_{2}+N_{2}-N_{1} \text {, } \\
& \Gamma(e)=\alpha_{1}+\frac{1}{2} \alpha_{2}^{\dagger} a, \\
& \Gamma(\bar{e})=\alpha_{2}+\frac{1}{2} \alpha_{1}^{\dagger} a, \\
& \Gamma(f)=\frac{1}{2}\left\{2 J_{1}-2 J_{2}-N_{a}\right\}_{q} a^{\dagger} \alpha_{2} \\
& -\frac{1}{4}\left(\left[2 J_{1}-2 J_{2}\right]_{q}+\left[2 J_{1}-2 J_{2}-2 N_{a}\right]_{q}\right) \alpha_{1}^{\dagger} \\
& +\frac{1}{4}\left\{2 J_{1}-2 J_{2}-2 N_{a}-1\right\}_{q} \alpha_{1}^{\dagger} N_{2}, \\
& \Gamma(\bar{f})=\frac{1}{2}\left\{2 J_{1}+2 J_{2}-N_{a}\right\}_{q} a^{\dagger} \alpha_{1} \\
& -\frac{1}{4}\left(\left[2 J_{1}+2 J_{2}\right]_{q}+\left[2 J_{1}+2 J_{2}-2 N_{a}\right]_{q}\right) \alpha_{2}^{\dagger} \\
& +\frac{1}{4}\left\{2 J_{1}+2 J_{2}-2 N_{a}-1\right\}_{q} \alpha_{2}^{\dagger} N_{1}, \\
& \Gamma(E)=a, \\
& \Gamma(F)=\frac{1}{4}\left(q-q^{-1}\right)^{2}\left[4 J_{2}\right]_{q}\left(a^{\dagger}\right)^{2} \alpha_{1} \alpha_{2} \\
& +\frac{1}{4}\left(\left[4 J_{1}-3 N_{a}+2\right]_{q}+\left[4 J_{1}-N_{a}\right]_{q}\right) a^{\dagger}+\frac{1}{8}\left\{4 J_{2}\right\}_{q}\left\{N_{a}-1\right\}_{q} a^{\dagger} \\
& -\frac{1}{4}\left\{4 J_{1}-3 N_{a}+1\right\}_{q} a^{\dagger} N_{1}-\frac{1}{8}\left\{4 J_{2}\right\}_{q}\left\{N_{a}-1\right\}_{q} a^{\dagger} N_{1} \\
& -\frac{1}{4}\left\{4 J_{1}-3 N_{a}+1\right\}_{q} a^{\dagger} N_{2}-\frac{1}{8}\left\{4 J_{2}\right\}_{q}\left\{N_{a}-1\right\}_{q} a^{\dagger} N_{2} \\
& -\frac{1}{8}\left(\left\{4 J_{2}-N_{a}-1\right\}_{q}+\left\{4 J_{2}+N_{a}+1\right\}_{q}\right. \\
& \left.-\left\{4 J_{2}-N_{a}+1\right\}_{q}-\left\{4 J_{2}+N_{a}-1\right\}_{q}\right) N_{1} N_{2} a^{\dagger} \\
& -\frac{1}{4}\left(\left\{4 J_{1}-3 N_{a}-1\right\}_{q}-\left\{4 J_{1}-3 N_{a}+1\right\}_{q}\right) N_{1} N_{2} a^{\dagger} \\
& +\frac{1}{16}\left(\left[4 J_{2}-2 N_{a}-1\right]_{q}+\left[4 J_{2}+2 N_{a}+1\right]_{q}+\left[4 J_{2}-1\right]_{q}+\left[4 J_{2}+1\right]_{q}\right) \alpha_{1}^{\dagger} \alpha_{2}^{\dagger} \text {. }
\end{aligned}
$$

Using expression in Section I, we prove that operators in (31) also satisfy super commuatation relations as in (20). It means that mapping (29) does perform a boson-fermion realization of quantum super group $U_{q}[\operatorname{osp}(2 / 2)]$. Basically, we can derive basis vector in representation space via (28). However, this selection is not optimal to create physical system and thus we will show possible solution in separated works. 


\section{CONCLUSION}

This work is still far from being finished but the present results give us a good star to a more complete construction of representations in a coherent state basis of important quantum superalgebras, in particular, $U_{q}[\operatorname{osp}(2 / 2)]$ which is a quantum deformation ana$\log$ of the superalgebra $\operatorname{osp}(2 / 2)$ which has been intensively investigated and applied to different physics models.

\section{ACKNOWLEDGMENT}

The authors would like to thank Le Ba Nam and Nguyen Thi Hong Van for fruitful discussions and participation in this series of research. One of the authors (N.A.K.) would like to thank P. Aurenche and F. Boudjema for kind hospitality at LAPTH, Annecy, France. This work was supported by the Institute of Physics and the Vietnam Academy of Science and Technology (VAST) under grant VAST.HTQT.PHAP.04/2012-2013.

\section{REFERENCES}

[1] E. Schrödinger, Naturwissenchaften 14 (1926) 664.

[2] A. Perelomov, Commun. Math. Phys. 26 (1972) 222.

[3] A. Perelomov, Generalized Coherent States and Their Applications, Springer-Verlag, Berlin, 1986.

[4] J. Klauder and B.-S. Skagerstam (eds), Coherent States, World Scientific, Singapore, 1985.

[5] J. Deenen and C. Quesne, J. Math. Phys. 25 (1984) 1638, 2354.

[6] D. Rowe, J. Math. Phys. 25 (1984) 2662.

[7] D. Rowe, G. Rosensteel and R. Carr, J. Phys. A: Math. Gen. 17 (1984) L399.

[8] D. Rowe, B. Wyboume and P. Butler, J. Phys. A: Math. Gen. 18 (1984) 939.

[9] D. Rowe, G. Rosensteel and R. Gilmore, J. Math. Phys. 26 (1985) 2787.

[10] I. Bars and M. Günaydin, Commun. Math. Phys. 91 (1983) 31.

[11] M. Günaydin, J. Math. Phys. 29 (1988) 1275.

[12] A. Belantekin, H. Schmitt and B. Barrett, J. Math. Phys. 29 (1988) 1643.

[13] R. Le Blanc and D. Rowe, J. Math. Phys. 30 (1989) 1415.

[14] C. Quesne, J. Phys. A: Math. Gen. 23 (1990) L43-L48.

[15] C. C. Gerry and P. L. Knight, Introductory Quantum Optics, Cambridge University Press, 2005.

[16] L. Mandel and E. Wolf, Optical Coherence and Quantum Optics, Cambridge University Press, 1995.

[17] K. Fujii, "Introduction to Coherent States and Quantum Information Theory", arXiv: quant$\mathrm{ph} / 0112090$.

[18] A. A. Tseytlin, "Semiclassical String and ADS/CFT" in String Theory: from Gauge Interactions to cosmology (NATO science series), eds. L. Baulieu et al, Springer, The Netherlands, 2006, p.265.

[19] V. G. M. Puletti and T. Mansson, "The dual string $\sigma$-model of the $S U_{q}(3)$ sector", arXiv: 1106.111

[20] M. Bojowald, Phys. Rev. D75 (2007) 123512.

[21] Y.-Z. Zhang and M. Gould, J. Math. Phys. 46 (2005) 013505; arXiv: math/0405043.

[22] Y.-Z. Zhang, arXiv:hep-th/0405066.

[23] Nguyen Anh Ky and Nguyen T. Hong Van, Adv. Nat. Sci. 5 (2004) 1; arXiv: math/0305195.

[24] Nguyen Cong Kien, Nguyen Anh Ky, Le Ba Nam and Nguyen Thi Hong Van, J. Math. Phys. 52 (2011) 123512; arXiv:1201.1206 [math-ph].

[25] Nguyen Cong Kien, Nguyen Anh Ky, Le Ba. Nam and Nguyen Thi Hong Van: "Coherent State Method in Constructing Representation of Super Quantum Groups", talk given at the "37th National Meeting on Theoretical Physics", Cua Lo, 6-9 August 2012.

[26] T. Deguchi, A. Fujii and K. Ito Phys. Lett. B238 (1990) 242.

Received 25 December 2012. 